\title{
Austrian Triad Complicated by Septic Arthritis and Aortic Root Abscess
}

\author{
Sandiya Bindroo ${ }^{1}$, Shafinaz Akhter ${ }^{2}$, Kshitij Thakur ${ }^{3}$, Charles Geller ${ }^{4}$ \\ 1. Internal Medicine, Crozer Chester Medical Center, Upland, USA 2. Infectious Disease, Penn Medicine \\ Chester County Hospital, Mount Laurel, USA 3. Internal Medicine, University of Kentucky College of \\ Medicine, Kentucky, USA 4. Cardiothoracic Surgery, Crozer Chester Medical Center, Upland, USA
}

$\square$ Corresponding author: Sandiya Bindroo, sandiya.bindroo@crozer.org

Disclosures can be found in Additional Information at the end of the article

\section{Abstract}

Austrian syndrome is a very rare manifestation of invasive Streptococcus pneumoniae infection comprising a triad of pneumonia, meningitis, and endocarditis, also known as Osler's triad. We herein report a rare case of Austrian syndrome further complicated by septic arthritis.

Categories: Cardiac/Thoracic/Vascular Surgery, Internal Medicine, Infectious Disease

Keywords: invasive pneumococcal disease, austrian syndrome, aortic root abscess, septic arthritis, aortic valve endocarditis

\section{Introduction}

Austrian syndrome is a rare manifestation of invasive Streptococcus pneumoniae infection. The earliest reports in the literature are from the pre-penicillin era. Currently, in an era of penicillin therapy and pneumococcal conjugate vaccination, diagnosing a disseminated pneumococcal infection is infrequent and rare [1-2].

Austrian syndrome comprises a triad of Streptococcus pneumoniae endocarditis, meningitis, and pneumonia, described by Osler in 1881 [1]. In 1957, Robert Austrian reported a total of eight cases, of which six died secondary to the rupture of the aortic valve [1].

We present a challenging case of Austrian syndrome further complicated by aortic root abscess and septic arthritis in a healthy adult.

\section{Case Presentation}

Received 06/02/2018

Review began 07/11/2018

Review ended 07/16/2018

Published 07/20/2018

\section{C) Copyright 2018}

Bindroo et al. This is an open access article distributed under the terms of the Creative Commons Attribution License CC-BY 3.0., which permits unrestricted use, distribution, and reproduction in any medium, provided the original author and source are credited.
A 60-year-old Caucasian male known to have a bicuspid aortic valve was admitted with a threeday history of cough, altered mental status, and left upper extremity weakness. He did not have any other significant medical or surgical history. At presentation, he was confused and afebrile. His Glasgow Coma Scale (GCS) score was 8/15 (E2V2M4), blood pressure was 124/70 mm Hg, respiratory rate was 22 breaths/min, and oxygen saturation was $86 \%$ at room air. He was intubated for airway protection and respiratory support. Cardiac auscultation revealed 3/6 systolic murmur in the right second intercostal space, whereas lung auscultation revealed left lower zone crepitation. The abdominal examination was normal; he did not have any scars to suggest splenectomy. A complete neurologic assessment was not feasible, as the patient was intubated.

The initial laboratory investigations showed a white blood cell count (WBC) of 14.7 K/UL 


\section{Cureus}

(reference range, 4.0 to $11.0 \mathrm{k} / \mathrm{UL}$ ) with $90.9 \%$ neutrophils, and the platelet count was $34 \mathrm{k} / \mathrm{UL}$ (reference range 145-400 k/UL). His erythrocyte sedimentation rate (ESR) was 71 (reference range, $0-22 \mathrm{~mm} / \mathrm{hr}$ for men). Blood cultures collected before the initiation of antibiotics grew Streptococcus pneumoniae, which was sensitive to ceftriaxone and penicillin. His urine was positive for the Streptococcus pneumoniae antigen.

A chest radiograph and computerized tomography (CT) scan of the head done on admission demonstrated areas of consolidation over his left lower zone and the dilation of the lateral and third ventricles, respectively (Figure $)$.

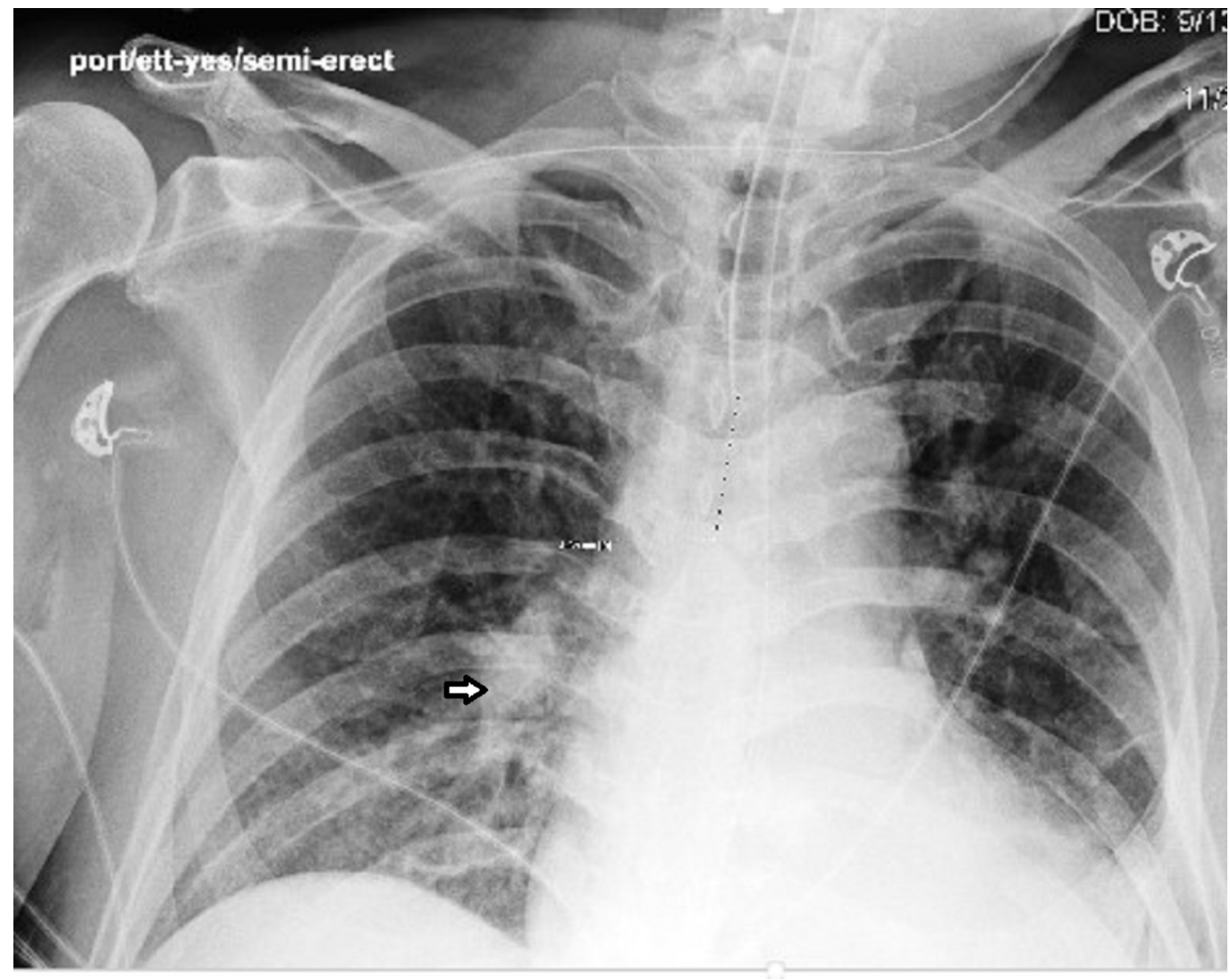

\section{FIGURE 1: Chest x-ray showing the presence of a left lower lobe infiltrate}

Treatment for bacterial meningitis was initiated with intravenous (IV) ceftriaxone, ampicillin, and dexamethasone empirically. Lumbar puncture was deferred due to the high risk of brain stem herniation secondary to hydrocephalus noted on head CT and high bleeding risk due to thrombocytopenia (platelet count $34 \mathrm{k} / \mathrm{UL}$ ). On day two of admission, magnetic resonance imaging (MRI) of the brain revealed scattered bilateral cerebral punctate infarcts suggestive of septic emboli along with hydrocephalus (Figure 2). A transesophageal echocardiogram (TEE) revealed caseous calcification of the bicuspid aortic valve and fusion of valve commissures with no vegetation. 


\section{Cureus}

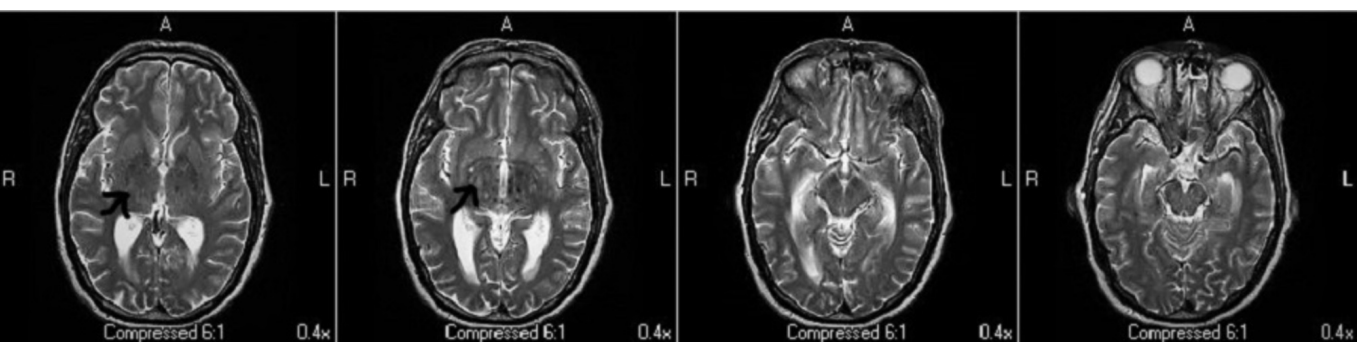

FIGURE 2: Magnetic resonance imaging (MRI) of the brain showing scattered punctate infarcts in the bilateral cerebrum and very mild hydrocephalus of the third and lateral ventricle

Over the course of hospitalization, his condition improved. Antibiotics were de-escalated. On day five of admission, he was successfully extubated. He remained cognitively impaired, which improved gradually.

On day nine of admission, he started complaining of right shoulder pain and had an elevation of leukocyte count (17.2 K/UL) with low-grade fever (99.9F). He underwent right shoulder arthroscopy and debridement for right shoulder septic arthritis. Gram staining of the synovial fluid showed few polymorphs, and the culture was negative. He was subsequently discharged home on a six-week course of IV ceftriaxone 2 g every 24 hours and a plan to get an aortic valve replacement.

Eight weeks after his initial presentation, he had a persistently elevated leukocyte count despite adequate medical management, prompting a treating physician to repeat the echocardiogram. A TEE was performed and documented aortic valve vegetations. Urgent cardiac surgery was performed.

Intraoperative findings include severe pericarditis, a bicuspid aortic valve, and multiple vegetations on the aortic valve leaflet, a $3 \times 3 \mathrm{~cm}$ aortic root abscess, and an approximately $10 \times 10 \mathrm{~cm}$ retro aortic abscess. He underwent a debridement of the retro-aortic abscess, pericardial patch repair, and aortic valve replacement. A valvular gram stain showed a few polymorphs and negative cultures.

He remained stable in the postoperative period and was subsequently discharged on intravenous penicillin to complete a course for another six weeks.

On a follow-up two months after discharge home, he remained stable with no significant anatomic or functional heart abnormalities and no neurological deficits.

\section{Discussion}

Streptococcus pneumoniae (S. pneumoniae) is a typical example of a highly invasive, Grampositive, extracellular bacterial pathogen [3]. In the antibiotic era, Streptococcus pneumoniae endocarditis is responsible for less than $3 \%$ of all cases of endocarditis in native valves. Pneumococcal endocarditis often causes extensive valve destruction and paravalvular involvement with a high mortality rate of $50 \%$ to $62 \%$ without surgical treatment [4].

Pneumococcal endocarditis is seen more commonly in middle-aged, debilitated men with a history of chronic alcoholism. Other risk factors for invasive pneumococcal infections include 
extremes of age; diabetes mellitus; chronic renal insufficiency; chronic liver disease; chronic pulmonary disease; anatomical or functional asplenia; tobacco abuse; certain ethnic groups, such as Alaskan natives; and other immunosuppressive conditions, such as human immunodeficiency virus (HIV) and multiple myeloma [5]. Interestingly, our case fitted in none of these predisposing conditions.

Classically, an invasive pneumococcal infection is meningitis and bacteremia and is defined as an infection confirmed by the isolation of Streptococcus pneumoniae from a normally sterile site (e.g., blood or cerebrospinal fluid but not sputum) [3]. In our case, the clinical diagnosis was confirmed by the positive blood culture and the presence of the Streptococcus pneumoniae antigen in urine. Though we were initially unable to perform a lumbar puncture secondary to increased bleeding risk, it was later on deemed unnecessary, as the patient improved clinically with medical management.

A clinical challenge exists in the early recognition of cardiac involvement, especially in patients who typically lack the peripheral features of infective endocarditis. A transesophageal echocardiogram (TEE) is significantly more sensitive than a transthoracic echocardiogram (TTE) in the diagnosis of endocarditis [6]. In our patient, the initial TEE was negative, delaying the confirmation of diagnosis further but high clinical suspicion guided the rest of the clinical course. This highlights the importance of careful daily review and clinical examination and maintaining a low threshold for suspecting endocarditis in a patient with ongoing sepsis.

Moreover, the involvement of different organs in patients with pneumococcal bacteremia also confounds the clinical presentation. It is important to be mindful of the potential for the spread of S. pneumoniae to other distant sites [7]. Septic arthritis is an uncommon complication of pneumococcal bacteremia. We herein report the presence of septic arthritis.

In a retrospective study of 80 cases of pneumococcal meningitis, only six patients developed endocarditis and death occurred in two patients due to cardiogenic shock [8]. However, Aronin et al. reported a $42 \%$ prevalence of Osler's triad in a review of pneumococcal endocarditis in the penicillin era, with a mortality rate greater than $50 \%$ [9].

Current literature on the treatment of Austrian syndrome emphasizes combined medical and surgical intervention. Due to the emergence of penicillin-resistant isolates, the initial use of combination antibiotics, such as a third-generation cephalosporin and vancomycin, are recommended. The antibiotic regimen can be modified once antimicrobial susceptibilities are available. Early surgical intervention in severe cases with invasive pneumococcal endocarditis decreases mortality by approximately $50 \%[4,10]$.

\section{Conclusions}

Given the rarity and high mortality associated with this disease, the report serves as a reminder of rare clinical illness and highlights the importance of early diagnosis and appropriate treatment to reduce the associated complications. Through this report, we wish to illustrate the importance of maintaining a high index of suspicion and pursuing this diagnosis in everyone who presents with severe sepsis and suspected pneumococcal infection, even if they do not have predisposing risk factors.

\section{Additional Information \\ Disclosures}

Human subjects: Consent was obtained by all participants in this study. Conflicts of interest: In compliance with the ICMJE uniform disclosure form, all authors declare the following: 
Payment/services info: All authors have declared that no financial support was received from any organization for the submitted work. Financial relationships: All authors have declared that they have no financial relationships at present or within the previous three years with any organizations that might have an interest in the submitted work. Other relationships: All authors have declared that there are no other relationships or activities that could appear to have influenced the submitted work.

\section{References}

1. Austrian R: Pneumococcal endocarditis, meningitis, and rupture of the aortic valve . Arch Intern Med. 1957, 99:539-544. doi:10.1001/archinte.1957.00260040039004

2. Olaison L, Pettersson G: Current best practices and guidelines Indications for surgical intervention in infective endocarditis. Infect Dis Clin North Am. 2002, 16:453-475.

10.1016/S0891-5520(01)00006-X

3. Henriques-Normark B, Tuomanen EI: The pneumococcus: epidemiology, microbiology, and pathogenesis. Cold Spring Harb Perspect Med. 2013, 3: 10.1101/cshperspect.a010215

4. Lefort A, Mainardi JL, Selton-Suty C, Casassus P, Guillevin L, Lortholary O: Streptococcus pneumoniae endocarditis in adults. A multicenter study in France in the era of penicillin resistance (1991-1998). The Pneumococcal Endocarditis Study Group. Medicine. 2000, 79:327337. 10.1097/00005792-200009000-00006

5. Yusuf J, Ahmad K, Mazumder S, Khouzam R: Streptococcus pneumoniae-associated pneumonia, meningitis, and endocarditis: a case of Austrian syndrome. J La State Med Soc. 2012, 164:324-326.

6. Shively BK, Gurule FT, Schiller NB, Roldan CA, Leggett JH, Schiller NB: Diagnostic value of transesophageal compared with transthoracic echocardiography in infective endocarditis. J Am Coll Cardiol. 1991, 18:391-397. 10.1016/0735-1097(91)90591-V

7. Milazzo L, Marchetti G, Negri C: A case of Austrian's syndrome with ocular involvement . Infection. 1999, 27:46-47. 10.1007/BF02565173

8. Auburtin M, Porcher R, Bruneel F, et al.: Pneumococcal meningitis in the intensive care unit: prognostic factors of clinical outcome in a series of 80 cases. Am J Respir Crit Care Med. 2002, 165:713-717. 10.1164/ajrccm.165.5.2105110

9. Aronin SI, Mukherjee SK, West JC, Cooney EL: Review of pneumococcal endocarditis in adults in the penicillin era. Clin Infect Dis. 1998, 26:165-171. 10.1086/516279

10. Bonow RO, Carabello BA, Chatterjee K, et al.: ACC/AHA 2006 guidelines for the management of patients with valvular heart disease: a report of the American College of Cardiology/American Heart Association Task Force on Practice Guidelines. J Am Coll Cardiol. 2006, 114:e84-e231. 10.1161/CIRCULATIONAHA.106.176857 